\title{
Micrometric Monodisperse Solid Foams as Complete Photonic Bandgap Materials
}

Ilham Maimouni $\uparrow^{1}$, Maryam Morvaridi $\dagger^{1}$, Maria Russo $\uparrow^{1,4}$, Gianluc Lui ${ }^{2}$, Konstantin Morozov $^{3}$, Janine Cossy ${ }^{4}$, Marian Florescu ${ }^{2}$, Matthieu Labousse ${ }^{* 5}$, Patrick Tabeling ${ }^{1}$

${ }^{1}$ Microfluidique, MEMS et Nanostructures, Institut Pierre-Gilles de Gennes, CNRS UMR 8231, ESPCI Paris and Paris Sciences et Lettres (PSL) Research University, 75005 Paris, France.

${ }^{2}$ Advanced Technology Institute and Department of Physics, University of Surrey, Guildford, Surrey GU2 7XH, United Kingdom

${ }^{3}$ Department of Chemical Engineering Technion - Israel Institute of Technology Haifa 3200003, Israel

${ }^{4}$ Molecular, Macromolecular Chemistry and Materials, ESPCI Paris, CNRS, PSL University, 10 Rue Vauquelin, 75231 Paris Cedex 5, France

${ }^{5}$ Gulliver, CNRS UMR 7083, ESPCI Paris and Paris Sciences et Lettres (PSL) Research University, 75005 Paris France.

†These authors equally contributed.

Corresponding author: matthieu.labousse@espci.psl.eu 


\section{SI1. Geometrical characteristics of the used microfluidic devices}

Figure S. 1, Figure S. 2, Figure S. 3 show optical images of three different microfluidic chips used to produce the foam samples described in the main text. Table $\mathbf{S} 1$ illustrates the main dimensions (width $\mathrm{x}$ height) of the microfluidic devices used for obtaining foams showed in Figure 2a-d in the main text.

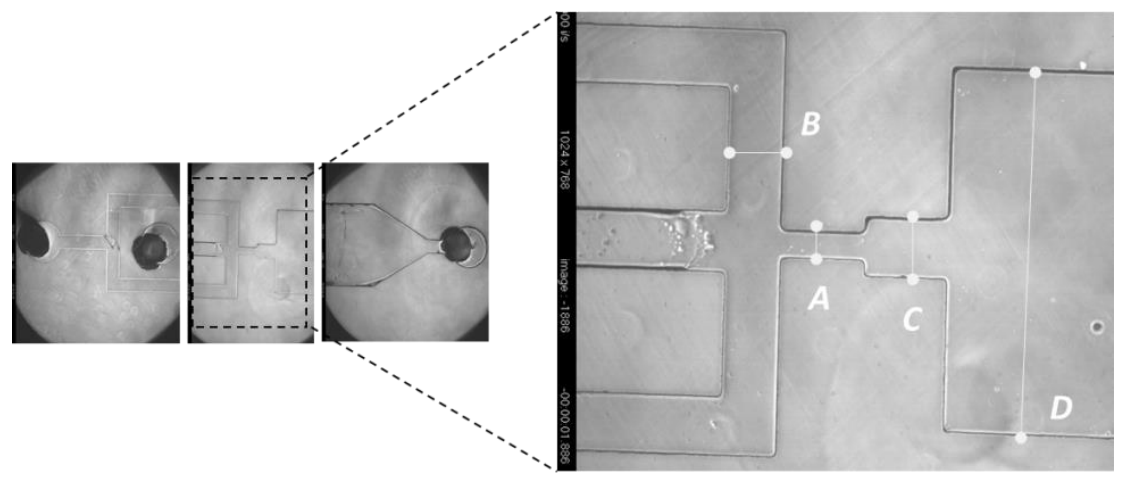

Figure S. 1 Optical image of the microfluidic device with a constriction A of $150 \mu \mathrm{m}$

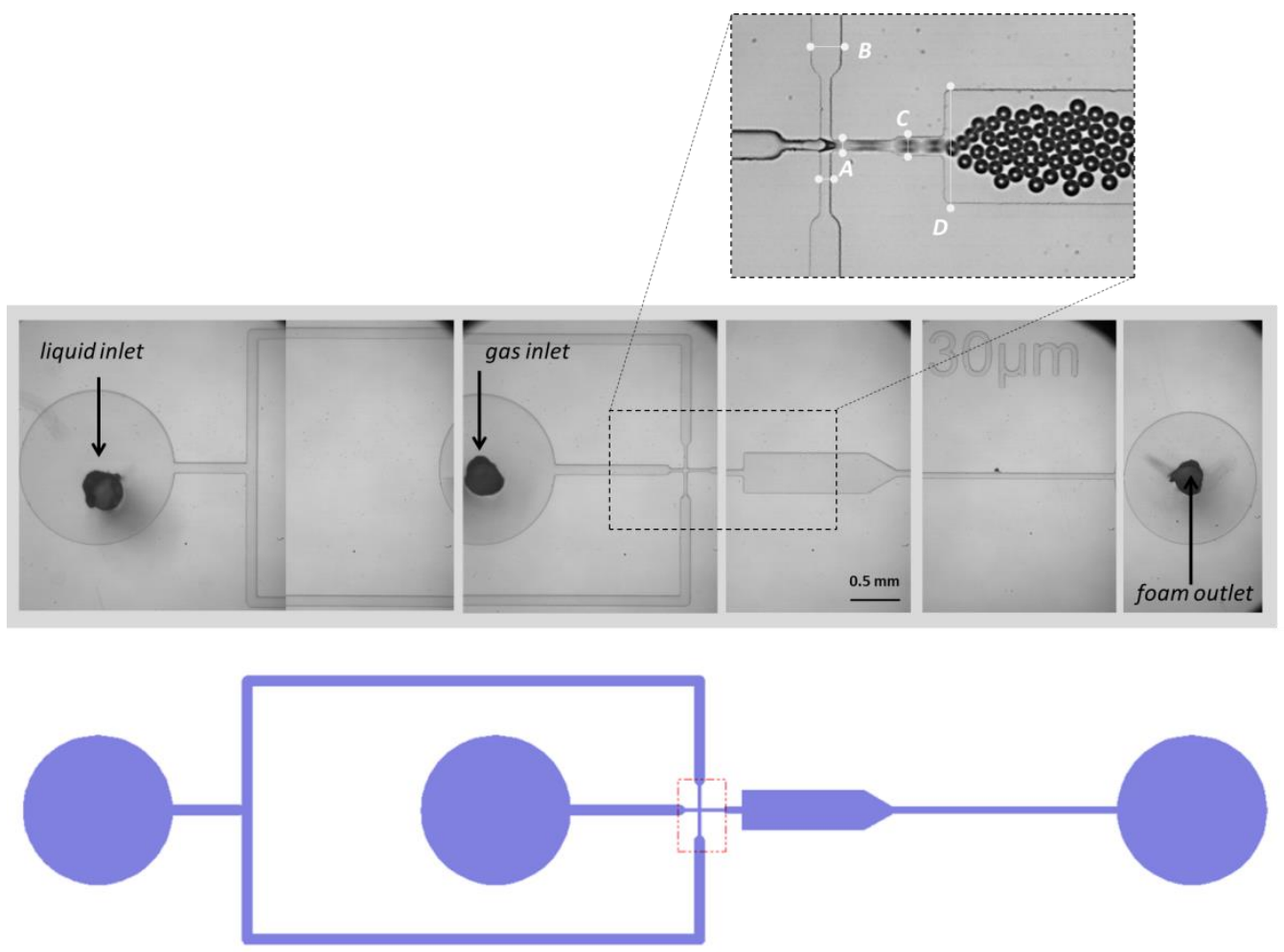

Figure S. 2 Optical image of the microfluidic device with a constriction A of $30 \mu \mathrm{m}$ 


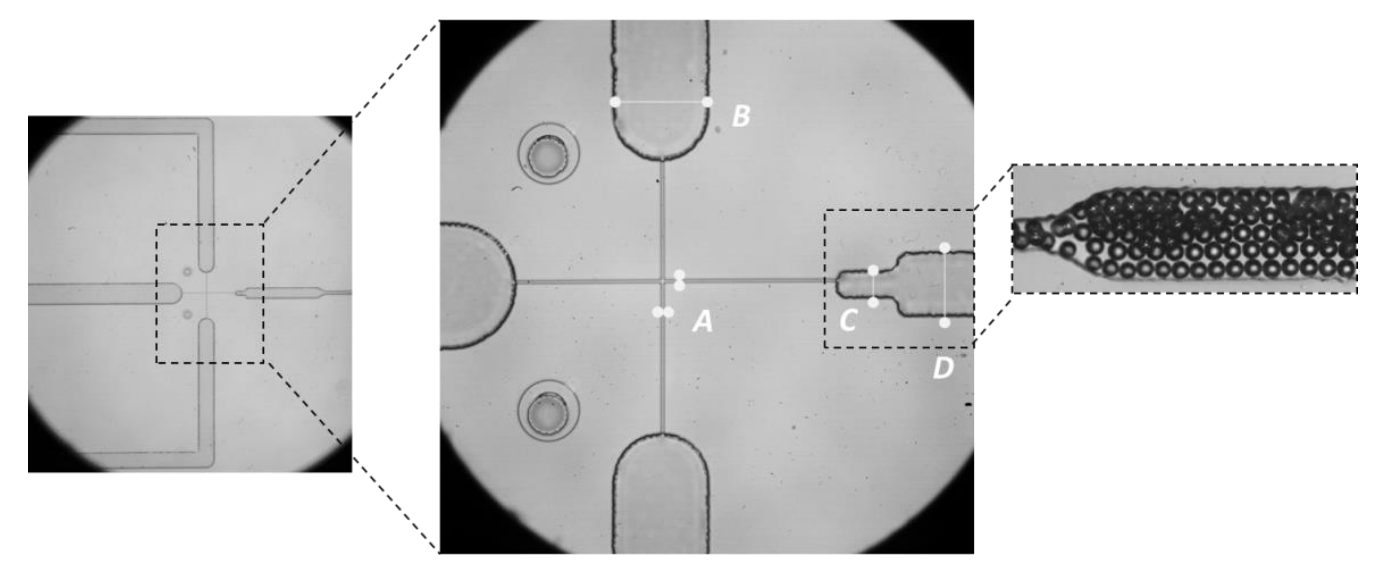

Figure S. 3 Optical image of the microfluidic device with a constriction A of $5 \mu \mathrm{m}$

Table S 1. Main dimensions (width $x$ height) of the used microfluidic devices

\begin{tabular}{|c|c|c|c|c|c|}
\hline $\begin{array}{c}\text { Bubbles' } \\
\text { Size }\end{array}$ & Chip & $\begin{array}{c}\left(\mathrm{A} \times \mathrm{h}_{\mathrm{A}}\right) \\
\mu \mathrm{m}\end{array}$ & $\begin{array}{c}\left(B \times h_{B}\right) \\
\mu m\end{array}$ & $\begin{array}{c}\left(\mathrm{C} \times \mathrm{h}_{\mathrm{C}}\right) \\
\mu \mathrm{m}\end{array}$ & $\begin{array}{c}\left(\mathrm{D} \times \mathrm{h}_{\mathrm{D}}\right) \\
\mu \mathrm{m}\end{array}$ \\
\hline $\begin{array}{l}400 \mu \mathrm{m} \\
\text { (Fig. 2a) }\end{array}$ & Fig. S1 & $150 \times 50$ & $300 \times 50$ & $300 \times 50$ & $200 \times 50$ \\
\hline $\begin{array}{c}60 \mu \mathrm{m} \\
\text { (Fig. 2b) }\end{array}$ & Fig. S2 & $30 \times 50$ & $100 \times 50$ & $60 \times 50$ & $400 \times 50$ \\
\hline $\begin{array}{l}10 \mu \mathrm{m} \\
\text { (Fig. 2c) }\end{array}$ & Fig. S3 & $5 \times 5$ & $100 \times 50$ & $30 \times 50$ & $60 \times 50$ \\
\hline $\begin{array}{c}5 \mu \mathrm{m} \\
\text { (Fig. 2d) }\end{array}$ & Fig. S3 & $5 \times 5$ & $100 \times 50$ & $30 \times 50$ & $60 \times 50$ \\
\hline
\end{tabular}

For the chip in Figure S. 3, filters were added to the inlets to avoid the introduction of dust (see Figure S. 4). 


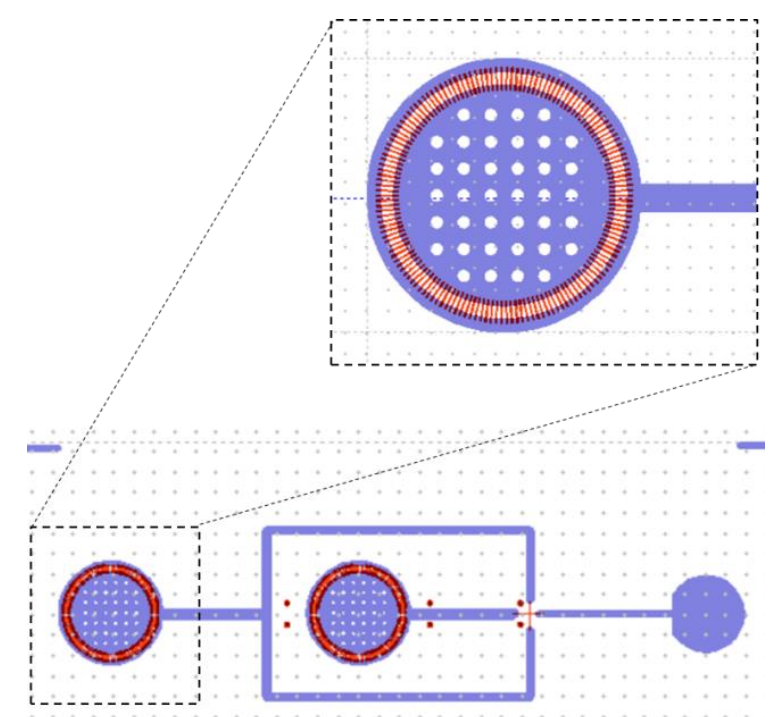

Figure S. 4 Schematic illustration of a FF microfluidic device where filters ( $4 \mu \mathrm{m}$ for a constriction of $5 \mu \mathrm{m}$ ) were added to the inlets. 


\section{SI2. Crosslinking methods}

In literature, when microfluidics is used for the production of 3D solid polymer foams, a cross-linking agent can be added at different steps of the production process following mainly two procedures:

- The cross-linker is added at the outlet of the device using for example a T junction inlet. In this case, the cross-linking begins after the foam formation (Figure S.5 A).

- The cross-linker is mixed directly with the liquid polymer phase. In this particular case, the cross-linking begins during the formation of the foam inside the microfluidic device (Figure S.5 B).
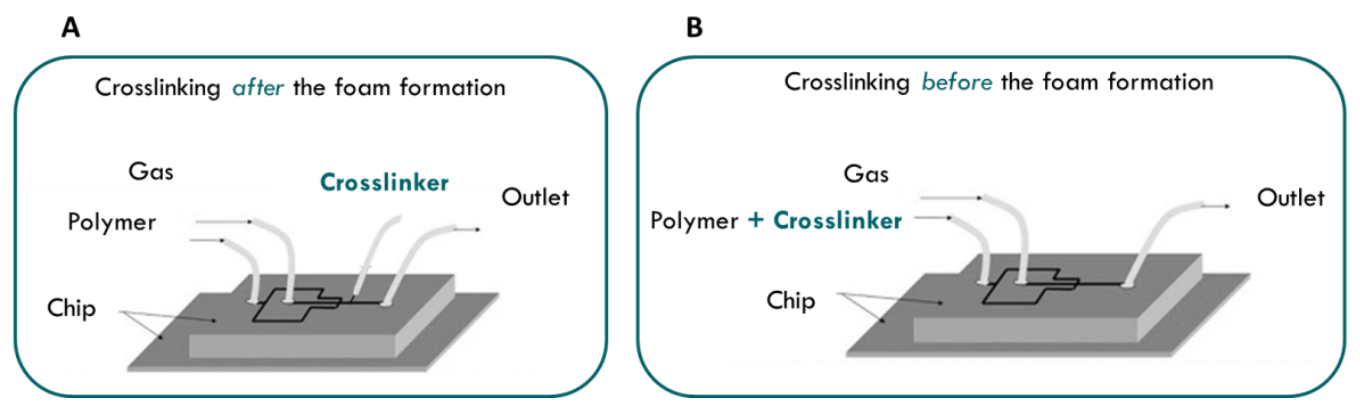

Figure S. 5 Crosslinking procedures in a Flow Focusing microfluidic geometry where the crosslinking reaction starts A) after and $B$ ) before the foam formation

In the first case (Figure S.5 A), since the cross-linker is added after the foam formation, it is not homogeneously distributed inside the foam. Consequently to the inhomogeneous cross-linking and the uncontrolled mechanical performances, bubbles sizes do not evolve uniformly and polydisperse foams are obtained.

In the second case (Figure S.5 B), the cross-linker is homogeneously distributed inside the foam. However, this process suffers from several limitations when the size of the microfluidic channels is smaller than $80 \mu \mathrm{m}$ or when a relatively large volume of foam (centimetric foam) has to be produced. Indeed, as the cross-linking begins in the micrometric channels, they get quickly clogged due to cross-linked polymer aggregates (Figure S. 6). The clogging disturbs the flows and bubbles get polydisperse. 


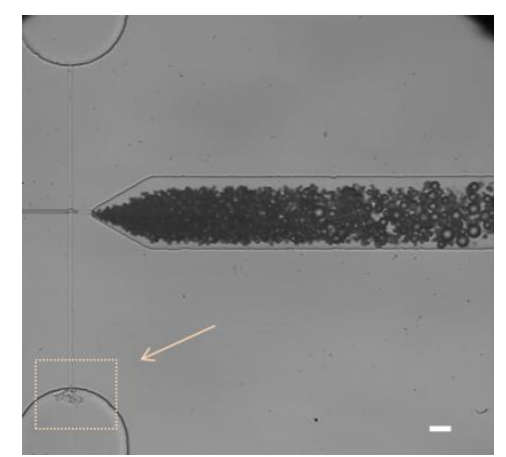

Figure S. 6 Foam generation following procedure B. Microfluidic channel get clogged and bubbles are polydisperse. Scale bar $30 \mu \mathrm{m}$

In summary, following one of these two procedures, the designing of 3D polymer solid foams, with a highly controlled pore size, especially lower than $80 \mu \mathrm{m}$, cannot be achieved.

Consequently, the process described in the main text resolves these problems and allows for the first time to fabricate self-assembled solid foams that exhibit a complete photonic bandgap. More generally, it pushes the existing limits of solid foam pore sizes (down to $5 \mu \mathrm{m}$ ) still keeping high control of the scaffold structure, which can be very interesting for broader applications like tissue engineering. 


\section{SI3. Layer-by-layer solidification process for foam sample}

In order to minimize the structure evolution during the solidification process due to the gravityinduced-drainage, the foam is generated following a "layer-by-layer" process: the foam flows through the outlet tube and forms a first layer which is collected on a glass slide. The height of this layer is kept smaller than the capillary length of the liquid phase (around 1- $3 \mathrm{~mm}$ ) in order to limit the gravity drainage. The layer is then sent to crosslinking in an oven at $70^{\circ} \mathrm{C}$ for $1 \mathrm{~min}$. Right after, another layer is added above and the same operation is reiterated until the desired height of the sample is reached. Following this procedure and using one microfluidic generator (one nozzle), a crosslinked foam sample of $2 \mathrm{~cm}^{3}$ (with pore sizes $=100 \mu \mathrm{m}$ ) can be produced in 1 hour. The parallelization of 5-10 microfluidic chips would allow to multiply the produced volume accordingly.

Figure S. 7 sums up the key steps that the foam goes through before we get the final samples.

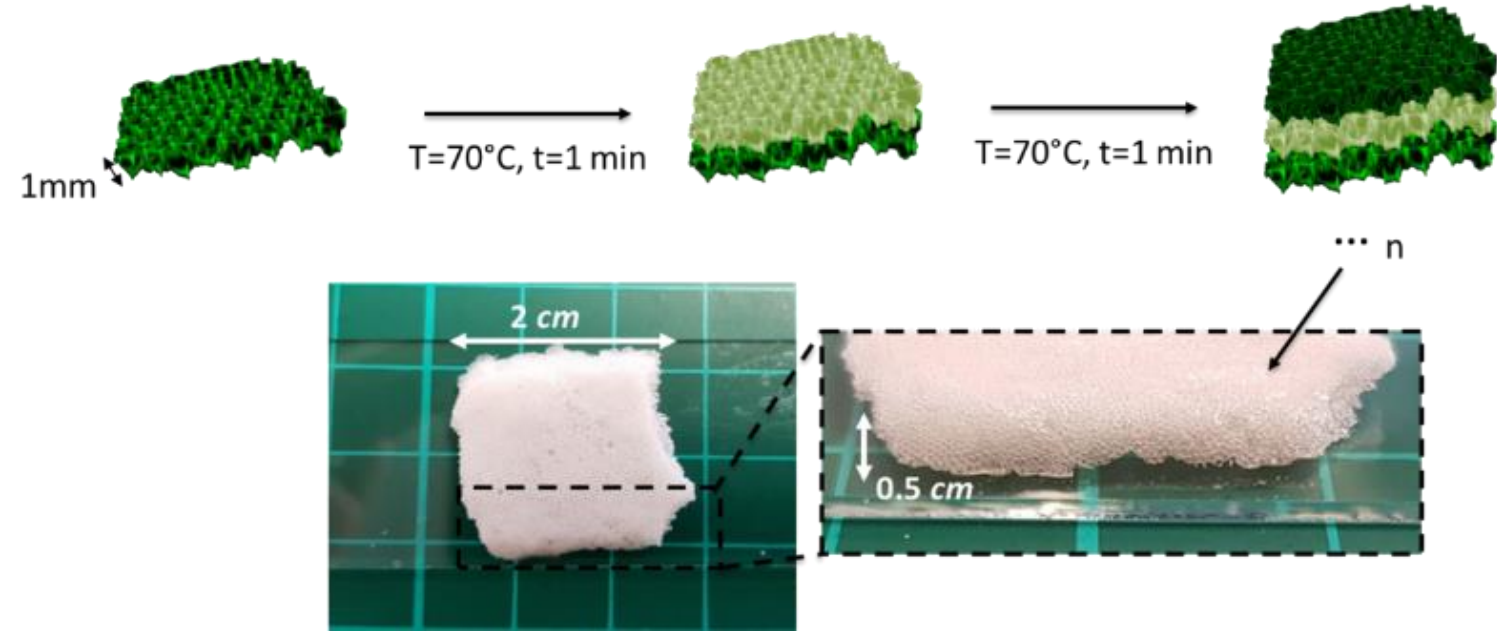

Figure S. 7 Layer-by-layer solidification process for foam samples higher than 1-3 $\mathrm{mm}$ : schematic representation of the different steps and a picture of an obtained chitosan foam (pore size 100 $\mu \mathrm{m}$ ) 


\section{S14. Plateau Border thickness profile analysis}

An image analysis of the Plateau Border (PB) diameter variation along its longitudinal axis ( $\mathrm{x}$ axis) was performed using Matlab. First, the confocal image of a representative sample of a chitosan foam (pore size $50 \mu \mathrm{m}$ ) was thresholded (Figure S. 8a). Then, the binary image was discretized into equally large strips along the $x$ axis and the white area of each strip was calculated as a function of its coordinate. The graph represents the variation of the diameter of the PB along its $x$ axis (Figure $S$. $8 b)$. The results show that the plateau borders get thicker in the close vicinity of the nodes but are of a constant thickness in most of the central region (around two-third of the PB length).

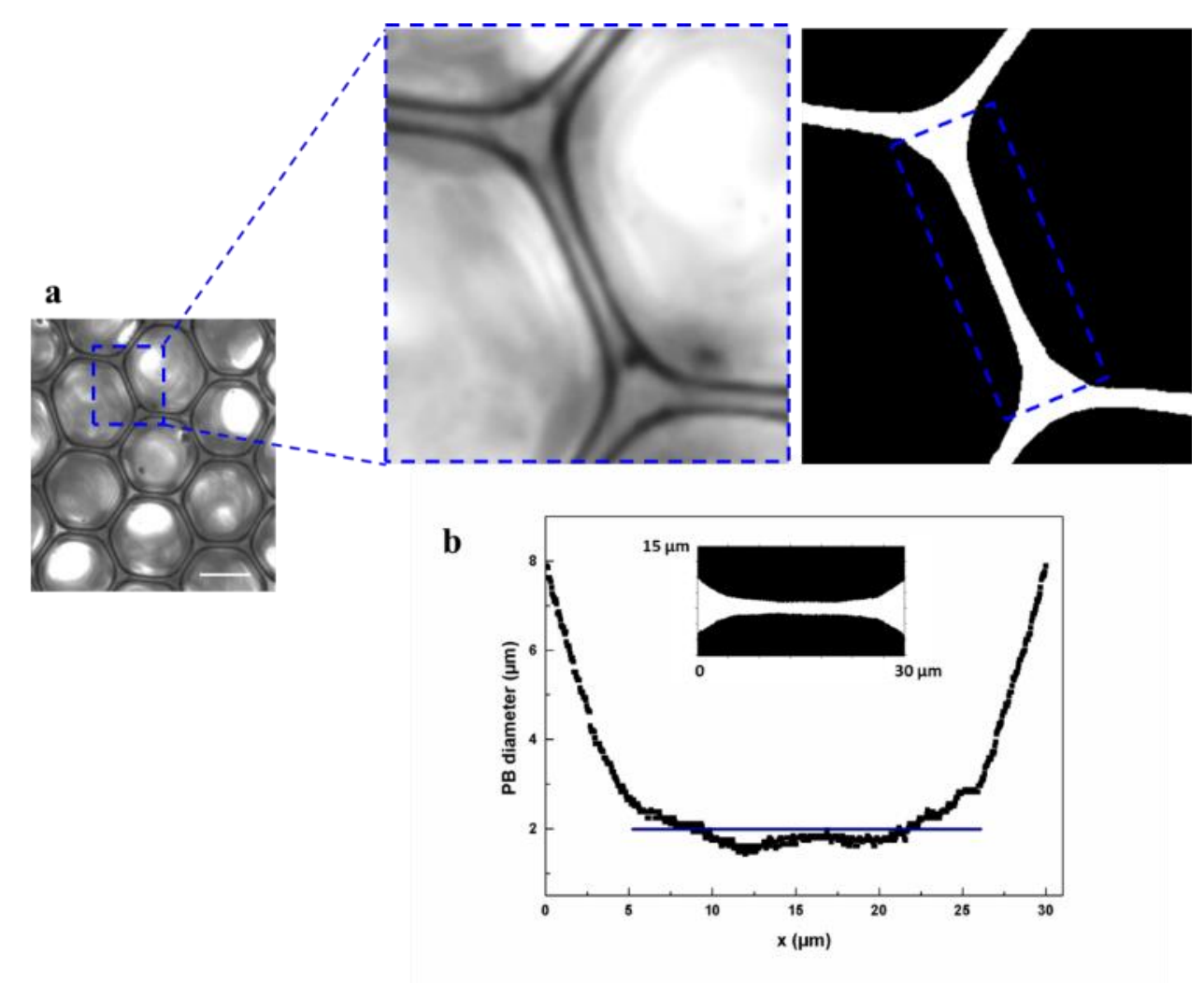

Figure S. 8 Plateau Border (PB) profile analysis. a) Confocal bright-field image of chitosan solid foam with zoom on a Plateau Border and a contour profile. Scale is $30 \mu \mathrm{m} ; b)$ Characteristic variation of PB diameter along its length. 


\section{SI5. Coating of the 3D solid polymer structure}

Two different coating techniques have been explored: dip-coating and sputtering.

\section{- Dip coating}

Few tests were conducted to coat chitosan solid foams with SiC (silicon carbide) (high refractive index in $\mathrm{THz}$ ) and titanium dioxide $\left(\mathrm{TiO}_{2}\right)$ (high refractive index in visible) nanoparticles (NPs). These NPs can be grafted onto chitosan due to electrostatic interactions.

First, $\mathrm{SiC}$ and $\mathrm{TiO}_{2} \mathrm{NPs}$ were dispersed, by using an ultrasonic bath for $20 \mathrm{~min}$, in two different precursor solutions:

- in water

- in aqueous solutions containing different percentages of ethanol (from $50 \% \mathrm{v} / \mathrm{v}$ to $90 \% \mathrm{v} / \mathrm{v}$ ) After this step, the foam sample was immersed into the precursor solution for a few minutes at a constant speed followed by a certain dwell time to leave sufficient interaction time between the substrate and the coating solution for complete wetting. After a few minutes, the sample was removed from the solution and was heated up to $70{ }^{\circ} \mathrm{C}$ for $1 \mathrm{~min}$ for solvent evaporation. The sample was then re-dipped into pure ethanol in an ultrasonic bath to only keep the NPS strongly attached to the structure. The sample was then dried at room temperature. The experiment was repeated several times until the desired deposit thickness was reached.

The results showed that, when the 3D solid foam was immersed in the ethanol free solution, the foam rapidly swells and collapses. When ethanol is added, it served as an anti-solvent for the chitosan limiting the swelling and the collapsing of the structure. In the meanwhile, water guarantees the preserving of a partial swelling of the chitosan meshes which promotes the incorporation of the NPs into the chitosan foam.

Figure S. $9 \mathrm{~A}$ and $\mathrm{B}$ show that, when the precursor solution was composed by $50 \% \mathrm{v} / \mathrm{v}$ of ethanol and $50 \% \mathrm{v} / \mathrm{v}$ of water, the coating of NPs was better (Figure S. 9B) than when the solution with $90 \% \mathrm{v} / \mathrm{v}$ of ethanol and $10 \% \mathrm{v} / \mathrm{v}$ of water was used (Figure S. 9A). Qualitatively, a larger surface of the Plateau borders was covered and a thicker layer as obtained. 

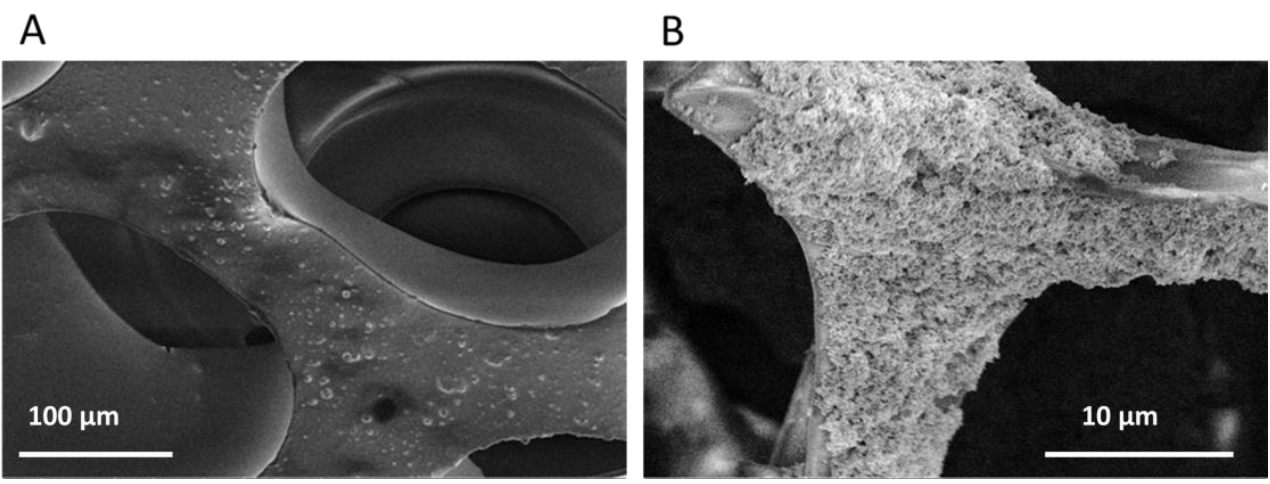

Figure S. 9 Dip-coating with two different SiC precursor solutions: A) 90\% ethanol and 10\% water; B) 50\% ethanol and 50\% water.

However, the surface is not completely covered and the thickness of the deposit is not uniform.

\section{- Sputtering (PVD method)}

Physical Vapor Deposition (PVD, Sputtering) has been tested. Shortly, this method involves the following steps:

- Creation of plasma (with the coating material ions and a gas, usually argon) under vacuum

- Exposure of the substrate to the plasma

- Thin layer deposition with a thickness that is time dependent $(\AA \AA-\mu \mathrm{m})$

In order to estimate the thickness of the thin layer, the 3D foam was sliced and only one rod was visualized by using SEM. The coating of a rod of the 3D foam after sputtering of $\mathrm{TiO}_{2}$ for around $3 \mathrm{~h}$ is shown in Figure S. 10.

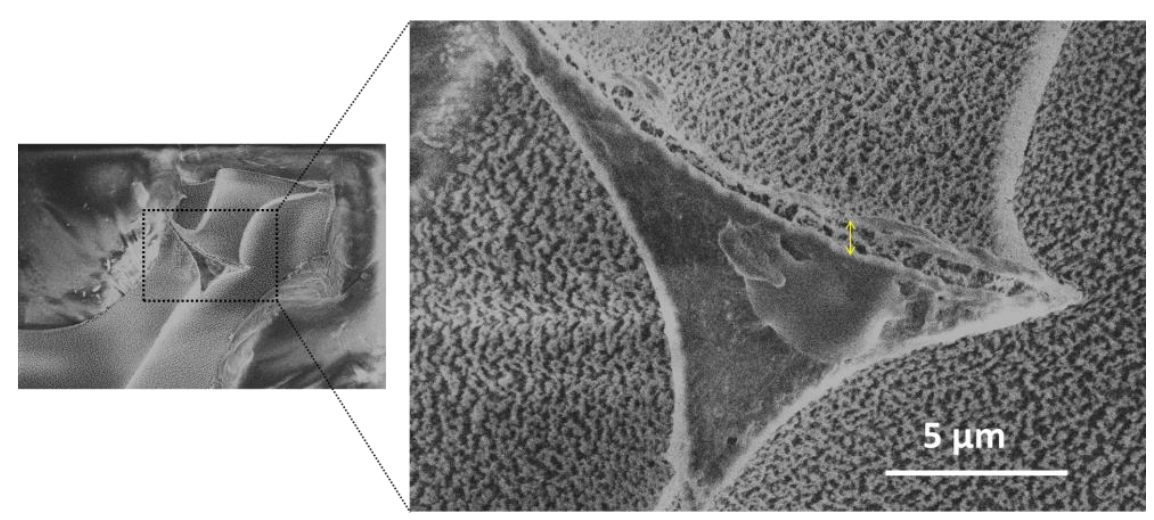

Figure S. 10 SEM images of the sputtering of Titanium dioxide for 3 hours on a 3D polymer foam.

Using SEM, we distinguish a thin layer of $\mathrm{TiO}_{2}$ which is $2-3 \mu \mathrm{m}$ high on top of the chitosan core. Preliminary results show that, unlike with dip-coating, the chitosan foam surface is completely 
covered. The coating surface appears to be grainy (with peaks and holes), however the holes spacing is more than one order of magnitude smaller than the rod typical size. In the mid and far infrared domain, the wavelength would be much larger than the characteristic surface rugosity so that the wavefield would be mainly insensitive to this sub-wavelength imperfection.

These considerations make of sputtering a serious coating option to consider for further investigations. 


\section{SI6. Band diagram using Comsol Multiphysics}

MPB numerical results have been confirmed by using an eigenmode decomposition technique in Comsol Multiphysics (Ver 5.4). In this approach, the electromagnetic modes of photonic crystal are computed through eigenvalue problem with finite element package and applying Bloch's theorem. This approach has been validated for the case for the case of an FCC lattice of close packed dielectric spheres in air and the resulting band diagrams were in full agreement with the solution reported in the literature. ${ }^{1}$ The important step to perform finite element method is assigning correct discretization. For 3D structures, the proper type of discretization is tetrahedral. The total volume of the system should be discretized in smaller elements. Finite element package Comsol Multiphysics is using proper optimization to get the solution converging.

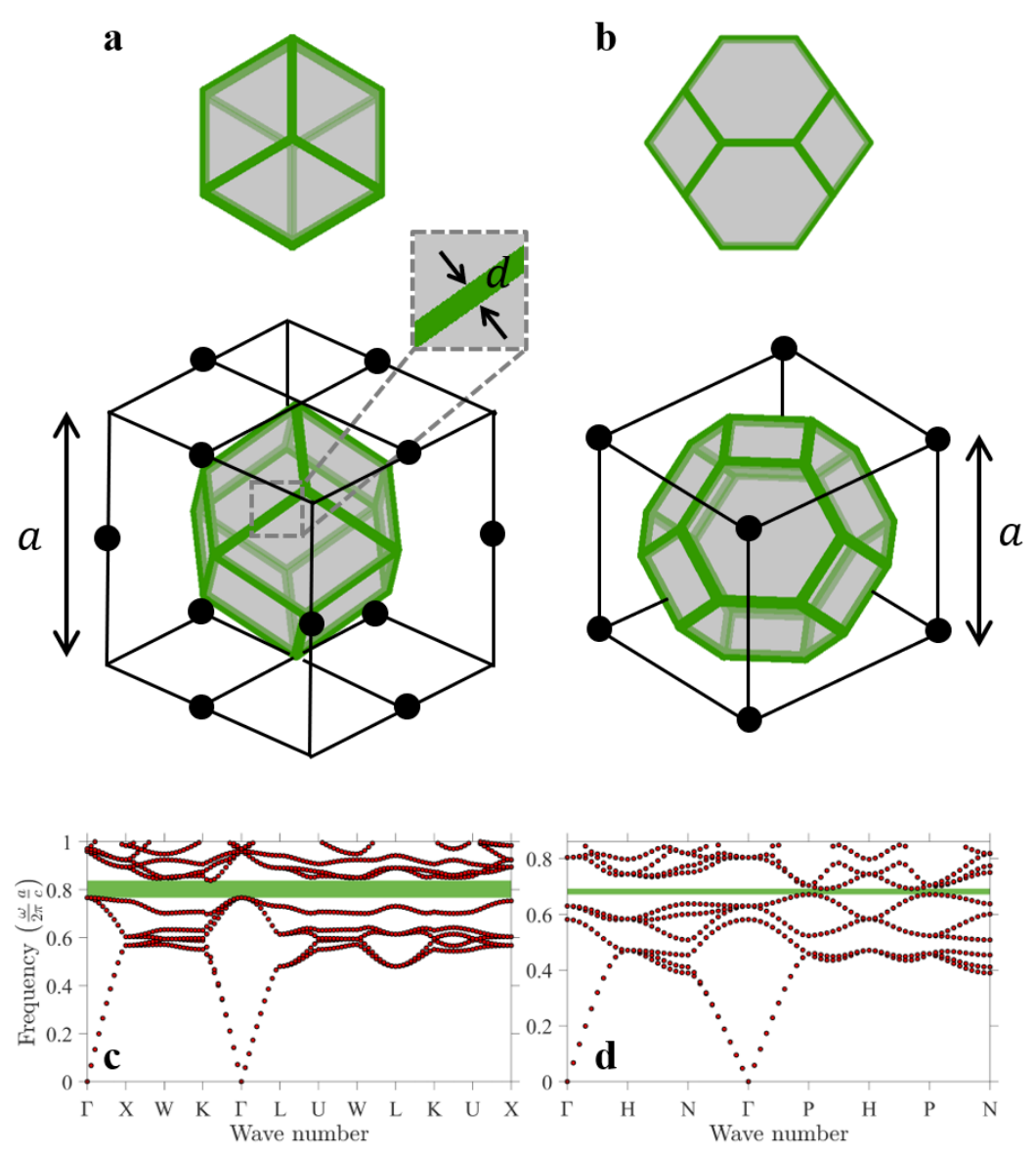

Figure S. 11 Solid foam structure and numerical photonic band diagram. a (respectively b) Geometry of the unit cell of the FCC-Kepler (resp. BCC-Kelvin) structure. Green rods of diameter d models a dielectric material while the remaining volume is air. $c$ (respectively d) numerical band diagram of the FCC (resp. BCC) solid foam for $a=115 \mu m, d=17.6 \mu m, \tilde{d}=0.153$ and $n=3.4$ (resp. $a=150 \mu m, d=30,27 \mu m, \tilde{d}=0.20$ and $n=3.4$ ). Green bands indicate the band gap opening separating the lower dielectric band from the upper air band. 


\section{SI7. Numerical field distribution analysis}

We illustrate in Figure S. 12 the spatial field mode intensities for the optimal structures $(n=3.4$, $\tilde{d}=0.153$ for the FCC structures and $n=3.4, \tilde{d}=0.20$ for the BCC structures) at the scale of one unit cell (Comsol simulations, Figure S. 12a,b,e and f), and for a finite sample consisting a few unit cells (MPB simulations, Figure S. 12c, d, g and h). We display the spatial field mode intensity of the dielectric and air band at the r-point (FCC structure, Figure S. 12a and b) and H-point (BCC, BCC structure, Figure S. $12 \mathrm{e}$ and f). Similarly, we display the extended spatial profile intensity for the lower photonic gap band edges ( $Г$-point for the FCC structure in Figure S. $12 \mathrm{~d}$ and the P-point for the BCC structure in Figure S. 12h) and for the upper photonic gap band edges (midway along the K- $\Gamma$ direction for the FCC structure in Figure S. 12c and midway along the P-H edge for the BCC structure in Figure S. 12h). Accordingly to the electromagnetism variational theorem ${ }^{1}$, the lower band gap edge has the field intensity concentrated into the region of larger dielectric constant (Figure S. 12b and d and Figure S. $12 \mathrm{f}$ and h) and conversely, the field intensity for the lower band edge is concentrated in the air fraction (Figure S. 12a and c and Figure S. 12e and g). This concentration of the field intensity into high/low index areas leads to the separation of the dielectric and air bands in the frequency domain and ultimately to the opening of the photonic band gap ${ }^{1}$. 

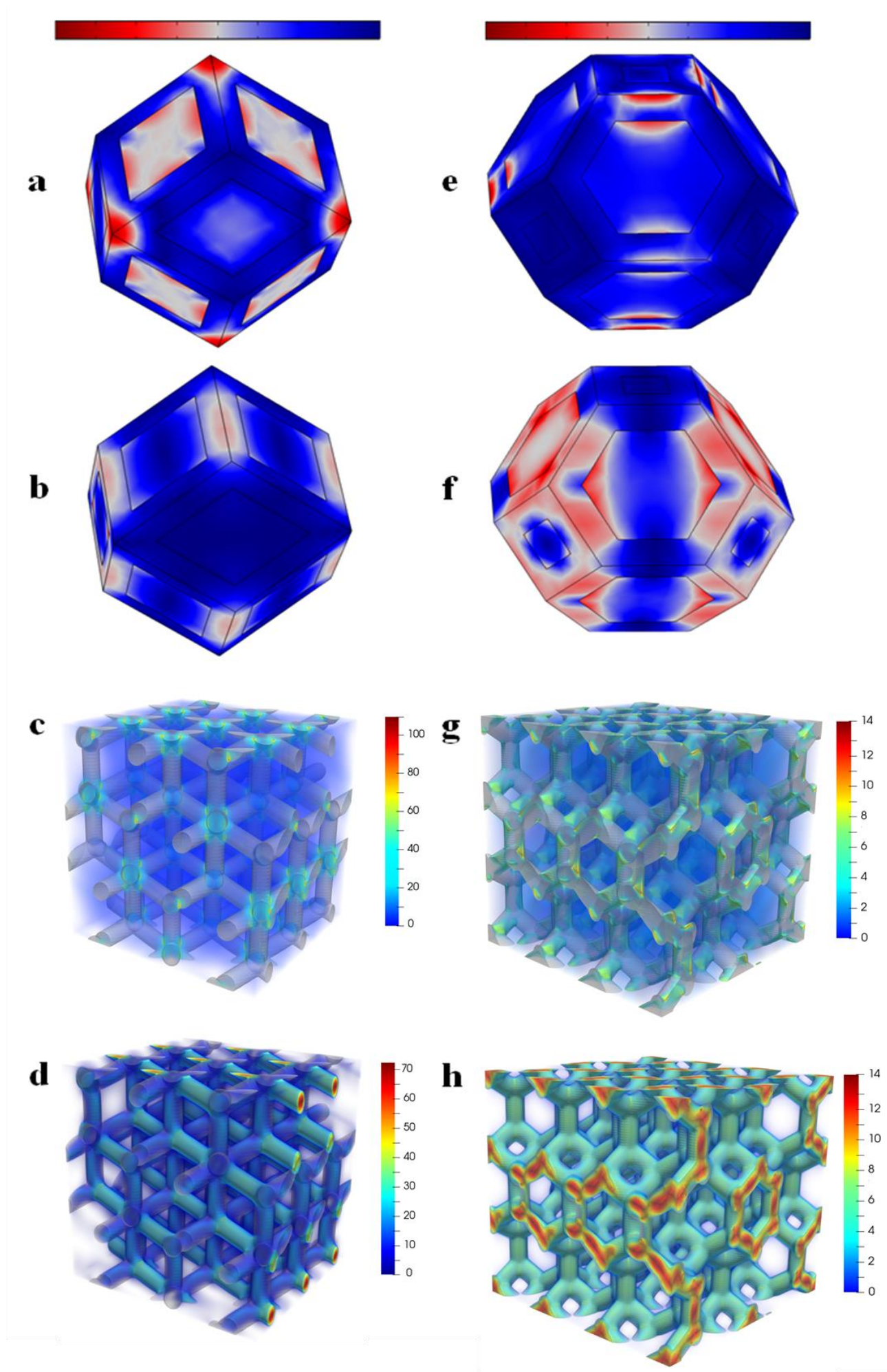

Figure S. 12 Analysis of field intensity when the gap band is open. $a$ and $b$ (resp e and f) Field intensity from Comsol calculations on a surface encapsulating the unit cell for FCC (resp. BCC) structure of geometry analogous to Figure S1 at the $\Gamma$ point of the Brillouin zone for FCC ( $H$ for BCC). The colormap accounts for the electric field norm intensity normalized by its maximum value (its significance being irrelevant for a linear system). a Air band of the FCC structure. $b$ Dielectric band of the FCC structure. $e$ Air band of the BCC structure. $f$ Dielectric band of the BCC structure. $c$ and $d$ (resp. $g$ and $h$ ) Field intensity from MPB calculations on a surface encapsulating the materials over several unit cells (resp. BCC). c Air band of the FCC structure (at its lower point). $d$ dielectric band of the FCC structure (at its upper point). $g$ Air band of the FCC structure (at its lower point). $h$ Dielectric band of the FCC structure (at its upper point). 


\section{SI8. Density of States Calculations}

Both, the band structure for the FCC and BCC optimal structures (displayed in Figure 3 of the main text) have the upper edge of the photonic band gap falling midway along Brillouin zone edges, ${ }^{2}$ typically not included in conventional band structure calculations. Here, we confirm our predictions for the band gap size of the optimal FCC and BCC structures against predictions from density of states (DOS) calculations. ${ }^{3,4}$ For DOS calculations, we use $32 \times 32 \times 32$ k-points distributed uniformly in the Brillouin zone. As shown in Figure S. 13, we obtain a nearly perfect agreement between band structure and DOS predictions for the spectral location and size of the photonic band gap.
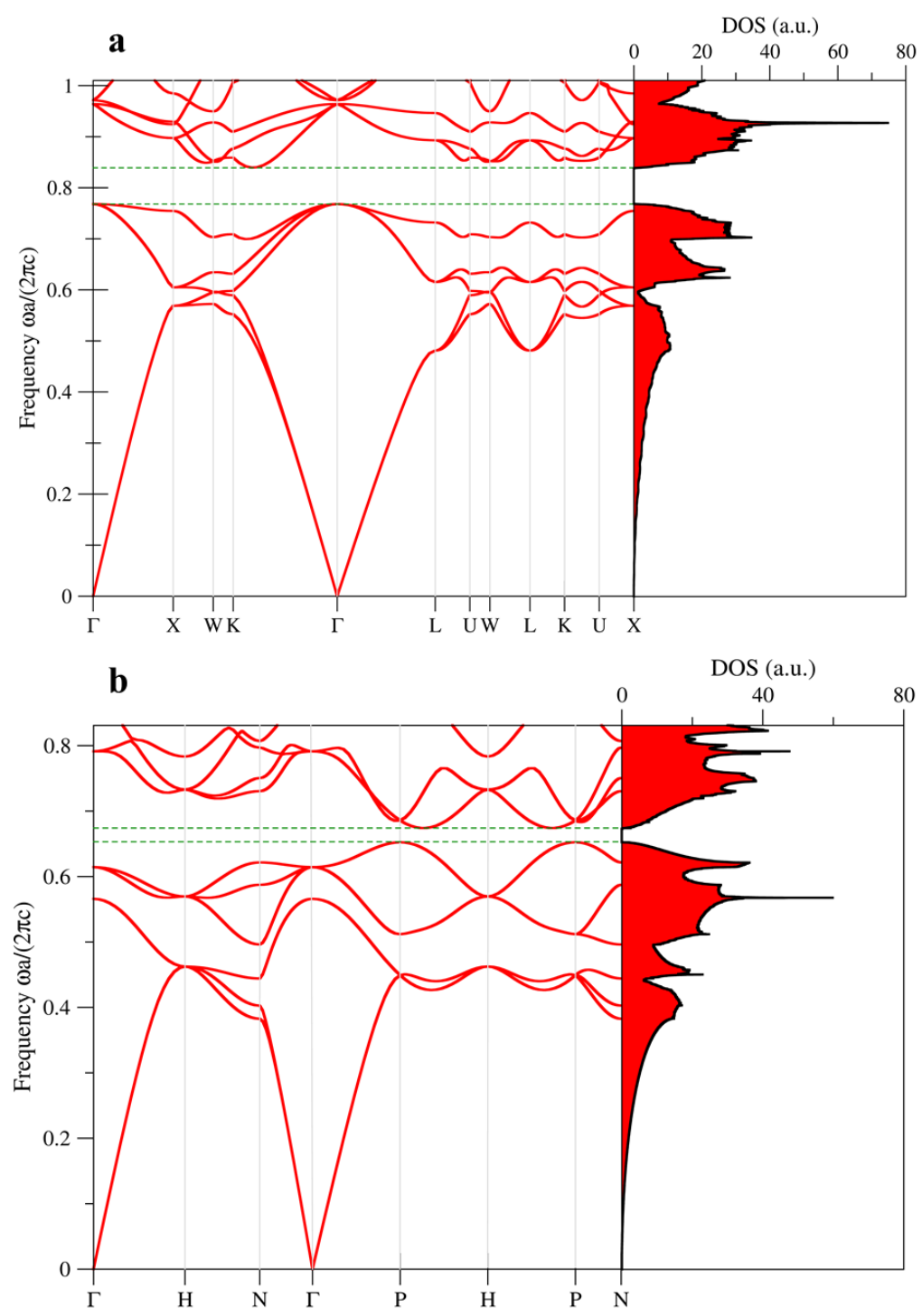

Figure S. 13 DOS calculations. $\boldsymbol{a}$ Band structure and DOS of the optimal FCC structure. $\boldsymbol{b}$ Band structure and DOS of the optimal BCC structure. 


\section{S19. Band diagram for closed cell foam}

The band structure has been simulated for closed cell foam structures (Figure S. 14). Closed cell foam structure is a unit cell of low refractive index material (air) surrounded by high refractive index material (dielectric material) (Figure S. 14a and b). Therefore, we have a closed unit cell of air inside a thin layer of dielectric material. The computation of the band structure confirms that closed cell foam structures do not show any PBG.
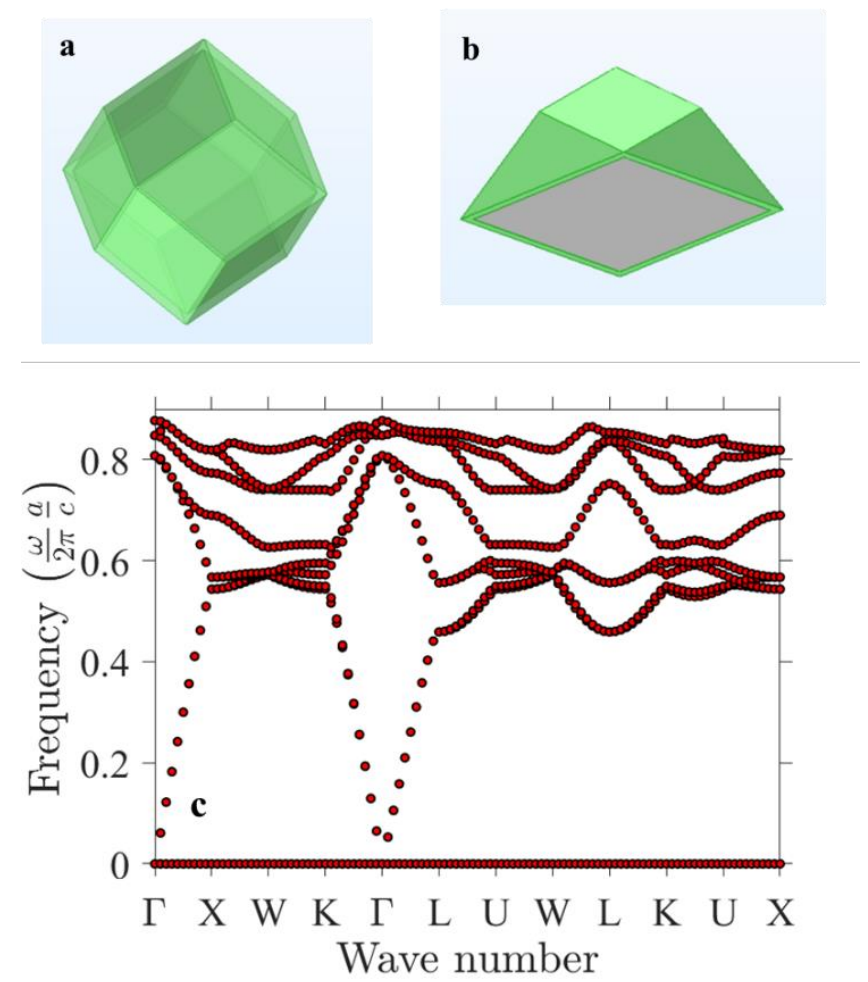

Figure S. 14 Closed cell foam geometry. a Full 3D view of the unit cell . $\boldsymbol{b}$ Half section of a foam unit cell. The dielectric material is shown in green and the air is presented in grey. $\mathbf{C}$ Photonic band diagram of closed cell-foam. 


\section{SI10. Distorted Network Decorations}

The simulations presented in this paper employ typical dry foam photonic network models (see for example ${ }^{5}$ ), in which the edges of the dry foam structures are homogeneously thickened into spherocylinders to provide a foam-like photonic network (as in Figure S. 15(b) below). Remarkably, regardless of the network topology, the largest band gap is obtained for a nearly identical filling fraction (for an index contrast of 3.4/1, the optimal filling fraction is about 20\%). Therefore, the precise structuring is less relevant if the symmetry of the overall structure is obeyed, and the optimal filling fraction is employed. To test this hypothesis, we have used a variety of network decorations shown in Figure S. 15. For each decoration and fixed index contrast, we have optimized over the geometrical characteristics of the decoration to obtain the largest gap. Overall, we have found very little variation between the values of the optimal gap obtained for various decorations (within few percent of each other). The largest gap for index contrast $1 / 3.4$ was obtained for the structure presented in Figure S. 16, with a value of $9.43 \%$ (the optimal gap for cylinder/sphero-cylinder decorations is $8.95 \%)$.
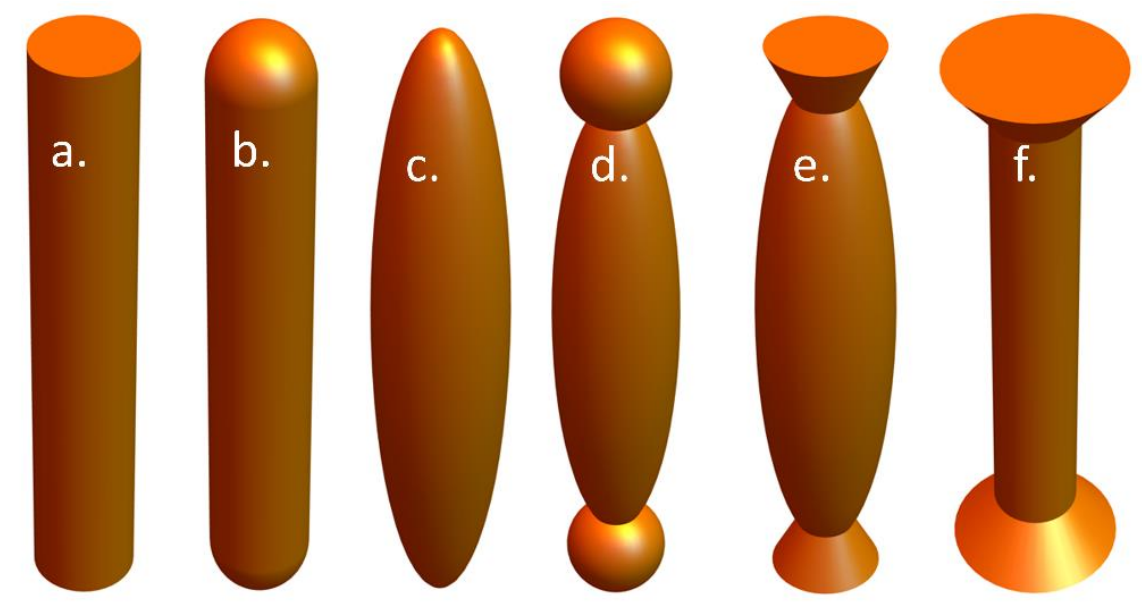

Figure S. 15 FCC network decorations. Various decorations employed in modelling FCC-foam-like structures. The photonic networks are generated by homogeneously thickening the edges of the foam into $\boldsymbol{a}$.) cylinders, b.) sphero-cylinders, c.) ellipsoids d.) sphero-ellipsoids e.) cone-ellipsoids or f.) cone-cylinders. 


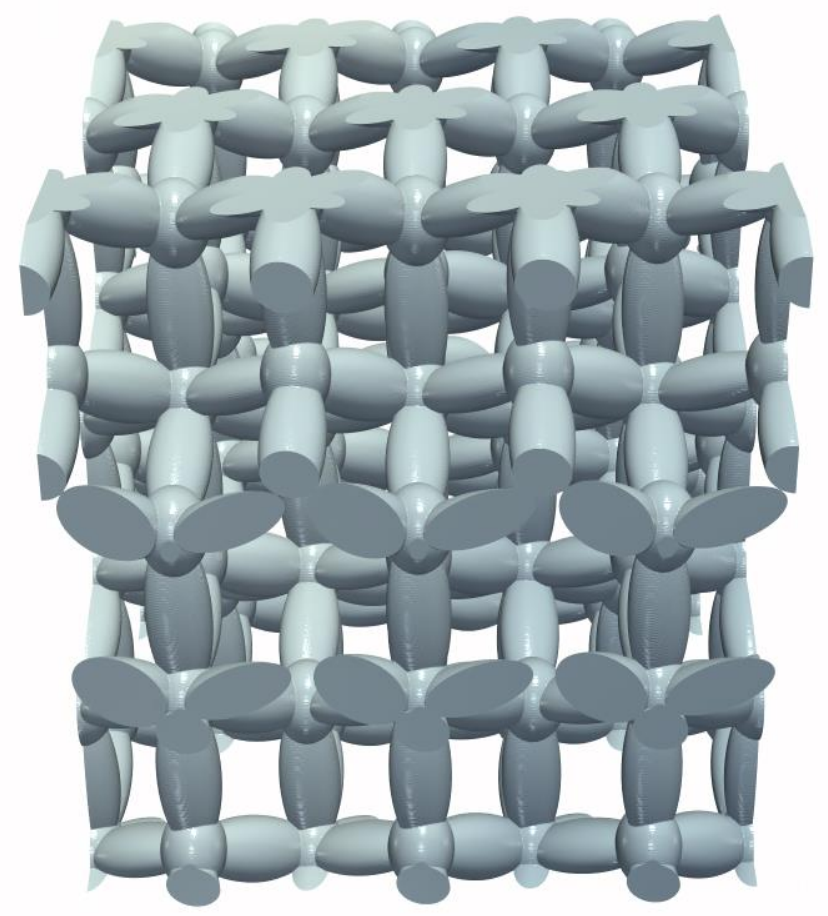

Figure S. 16 Optimal FCC-network structure. The structure presents a 9.43\% bandgap for an at index contrast of 3.4/1. The semi-axis lengths of the ellipsoid are given by $\alpha / a=0.433, \beta / a=0.165, \gamma / a=0.165$ and the radius of the sphere is $r / a=0.103$.

\section{References}

1. Joannopoulos JD, Johnson SG, Winn JN, Meade RD. Molding the Flow of Light. Princeton Univ Press, Princeton, NJ. 2008.

2. Setyavan W, Curtarolo S. High-throughput Electronic Band Structure Calculations: Challenges and Tools. Comput. 2010, 49, 299-312.

3. Johnson SG, Joannopoulos JD. Block-Iterative Frequency-Domain Methods for Maxwell's Equations in a Planewave Basis. Opt. 2001;8(3):173-190.

4. Liu B, Lu L, Johnson SG, Joannopoulos JD. Generalized Gilat-Raubenheimer Method for Density-ofstates Calculation in Photonic Crystals. J.Opt. 2018, 20, 044005.

5. Klatt MA, Steinhardt PJ, Torquato S. Phoamtonic Designs Yield Sizeable 3D Photonic Band Gaps. PNAS. 2019;116(47):23480-23486. 